\title{
UNA APROXIMACIÓN DE APLICACIÓN DE LA GESTIÓN DEL CONOCIMIENTO EN EL PODER JUDICIAL
}

\author{
Iris Estela Pacheco Huancas*
}

\begin{abstract}
Resumen
La gestión del conocimiento constituye en los tiempos actuales una poderosa herramienta de cambio e innovación en cualquier tipo de organización, tanto privada como pública.
\end{abstract}

En el presente artículo la gestión del conocimiento plantea la creación, organización, renovación, transmisión y utilización del conocimiento adquirido durante años de impartición de justicia por magistrados y personal jurisdiccional del Poder Judicial, en función a sus necesidades específicas.

La implementación de tecnologías de la información y la gestión del conocimiento en el ámbito judicial facilitaría y promovería el permanente aprendizaje dentro de la institución para lograr prontitud, certeza, predictibilidad en la solución de conflictos jurídicos.

Palabras clave: Gestión del Conocimiento - Factores claves - Metodología.

\begin{abstract}
At present, knowledge management is a powerful tool for change and innovation in any type of organization, whether private or public.

In this article, knowledge management proposes the creation, organization, renewal, transmission and use of the knowledge gained along the years by the justices and jurisdictional personnel of the Judiciary who have imparted justice based on their specific needs.

The implementation of information technology and knowledge management in the judicial environment would facilitate and promote permanent learning within the institution to achieve promptness, certainty and predictability in the solution of legal conflicts.
\end{abstract}

Key words: Knowledge management- Key factors - Methodology.

\section{Sumario}

1. Gestión del conocimiento y su importancia. 2. Concepto de conocimiento. 3. ¿Qué se entiende por gestión de conocimiento? 4. Objetivos de la gestión del conocimiento. 5. Factores claves de la gestión del conocimiento. 6. Campo de aplicación de la gestión del conocimiento. 7. Metodología para la gestión del conocimiento. 8. Conclusiones.

\footnotetext{
* Miembro del Consejo Consultivo del Centro de Investigaciones Judiciales del Poder Judicial de Perú y Jueza Superior Titular de la Corte Superior de Justicia de Lima Norte.
} 
Iris Estela Pacheco Huancas - Aplicación de la gestión

del conocimiento en el Poder Judicial

\section{GESTIÓN DEL CONOCIMIENTO Y SU IMPORTANCIA}

Las nuevas formas de economía en la aldea global han significado un quiebre respecto al cambio de paradigma del capitalismo industrial superado por la economía del conocimiento que tiene como base a los bienes intangibles que constituyen el capital intelectual, lo que en definitiva hoy en día permite un desarrollo sostenible de las organizaciones en el tiempo. Actualmente, los recursos intangibles son el eje central, factor de desarrollo de cualquier tipo de organización.

La densidad y atomización del conocimiento no le permite al ser humano manejarlo fácilmente y por ello requiere de un elemento clave para no ser "un sabio" sino saber ubicarlo cuando lo necesita, siendo el elemento clave la gestión del conocimiento en la llamada "sociedad del conocimiento".

Algunos autores consideran a la gestión del conocimiento una disciplina emergente. Su definición no es tan fácil, sin embargo, de la literatura revisada, existen elementos comunes, como la tendencia que el conocimiento se ha convertido en uno de los factores determinantes en el crecimiento de cualquier tipo de organización, no existiendo duda que es uno de los bienes intangibles más poderosos con que cuenta el ser humano, dando un valor económico a toda organización.

El Poder Judicial no es ajeno ni puede estar al margen de esta concepción ni de las nuevas tecnologías de la información y comunicación (NTIC) para su implementación. A diferencia de lo que sucede en el sector privado donde se busca el valor económico, en el ámbito público se genera un valor público. Hablando del órgano jurisdiccional, ese valor público se refiere al servicio de justicia que presta, en el que se busca generar eficacia, eficiencia y competitividad entre los integrantes de la organización.

El presente artículo tiene como objetivo plantear algunas pautas de aproximación de cómo puede ser aplicada la gestión del conocimiento en el Poder Judicial, que permita la creación, remoción, organización, compartición, uso y protección del conocimiento aprendido durante la impartición de justicia, de tal forma que genere su propia metodología de permanente aprendizaje entre los miembros de la organización, utilizando las herramientas tecnológicas para tener mayor certeza, facilidad y rapidez en la solución de conflictos jurídicos. 
Iris Estela Pacheco Huancas - Aplicación de la gestión del conocimiento en el Poder Judicial

\section{CONCEPTO DE CONOCIMIENTO}

"El conocimiento es una mezcla fluida y flexible de la experiencia, de los valores, de la información contextual y de la comprensión experta que proporciona un marco de trabajo para evaluar e incorporar nuevas experiencias e información. En definitiva, la información se transforma en conocimiento a través de las comparaciones con otras situaciones o informaciones, de las hipotéticas implicaciones de la información, de las conexiones con otros conocimientos (o partes del conocimiento) y de las conversaciones. Esta definición implica que el conocimiento en las organizaciones no solo se encuentra en los documentos o depósitos de información, sino también en las rutinas de las organizaciones, en sus procesos, prácticas y normas"1.

Muñoz y Seca (1997), citado por García Jiménez, afirma: “en una vertiente más ligada a la psicología y filosofía, se refieren al conocimiento como la capacidad de resolver un conjunto de problemas con una eficacia determinada. Al mismo tiempo, mencionan su volatilidad, ya que se ve modificado en función de los cambios que se producen en sus portadores a partir del aprendizaje, de la motivación y de la transferencia" ${ }^{2}$.

\section{3. ¿QUÉ SE ENTIENDE POR GESTIÓN DEL CONOCIMIENTO?}

Quienes definen a esta disciplina advierten cierta dificultad a la hora de dar una definición. La gestión del conocimiento es una disciplina emergente que se va afirmando con la aparición de nuevos paradigmas en los sistemas económicos nacionales e internacionales ${ }^{3}$.

Collison sostiene que la gestión del conocimiento versa sobre la captación, creación, depuración, uso compartido y puesta en práctica del know-how, "saber cómo"; en realidad una reducción de saber cómo, saber qué, saber quién, saber por qué y saber cuándo ${ }^{4}$.

Para Collison, la gestión del conocimiento no se refiere a la sabiduría de los libros ni a las mejores prácticas, sino a comunidades que mantienen vivo el know-how de una especialidad compartiendo lo que saben, construyendo a partir de ello y adaptándolo a sus necesidades. No es una instantánea de lo que se sabe en un momento determinado sino un conjunto de know-how que evoluciona y se pone al día gracias a la gente que lo utiliza regularmente ${ }^{5}$.

Francesc Solé y Martha Olea establecen una distinción sustancial entre recursos intangibles y tangibles. “Los recursos intangibles de una organización 
crecen generalmente por flujos de información o de conocimiento y los recursos tangibles crecen por flujos de dinero. Así por ejemplo, para adquirir maquinaria basta con tener dinero o crédito, mientras que para añadir nuevas características a un producto que satisfagan a los eventuales clientes se precisa de información y conocimiento". "[...] parece razonable afirmar que por mucho dinero que tenga una organización, si no es capaz de gestionar sus flujos de información y de conocimiento adecuadamente, será incapaz de construir un stock suficiente de recursos intangibles como para mantenerse competitiva"6.

Solé y Olea vinculan información y conocimiento. Para ellos, la gestión del conocimiento es la manera como la información se procesa con el fin de que se convierta en conocimiento. Así, las organizaciones deben averiguar cómo, cuándo y por qué se produce ese cambio, y, así, establecer patrones de comportamiento o pautas que relacionen esa transformación de información en conocimiento de los recursos intangibles de la organización ${ }^{7}$.

Estos autores, tomando como fuente el I Congreso Internacional de Gestión del Conocimiento y Capital Intelectual, realizado en mayo de 2000 en Madrid, señalan que durante la última década de los noventa se ha ido desarrollando una teoría sobre el capital intelectual y la gestión del conocimiento y, sin embargo, es general que ambos conceptos, o bien no se definen o bien al hacerse ambiguamente resulta difícil reconocer las líneas de separación entre ambos, mas aún, si aparecen sinónimos como recursos intangibles, recursos invisibles, etc., lo que dificulta su delimitación. Tratando de aclarar sus fronteras, sostienen que el capital intelectual se define como el "conjunto de recursos intangibles de la organización que tienen la capacidad de generar valor ya sea en el presente, ya sea en el futuro" ${ }^{\prime \prime}$.

Entre las formas de clasificación de intangibles Edvinsson y Malone (1999) ${ }^{9}$ distinguen las categorías de capital humano, capital estructural y capital relacional, las que desarrollando aplicamos al ámbito judicial:

a) El capital humano está formado por los recursos intangibles capaces de generar valor que residen en las personas: sus habilidades, conocimientos y capacidades. Este es el dominio del conocimiento y del aprendizaje de la persona y consecuentemente de la formación.

En esta categoría, debemos afirmar que el Poder Judicial cuenta con una reserva de conocimientos, capacidades y experiencias de los magistrados y personal, en el aprendizaje diario de construcción de jurisprudencia al 
resolver conflictos jurídicos comunes y complejos, siendo los primeros los que deben ser utilizados para elaborar una base de datos de casos tipo, utilizando para ello criterios propios de cada área del derecho y en cuya elaboración deben participar todos los magistrados puesto que ellos serán los primeros usuarios del sistema. Si bien ya se ha dado inicio a la sistematización de la jurisprudencia en la institución, sin embargo, esta aún no responde a un proyecto de gestión de conocimiento.

b) El capital estructural está formado por los recursos intangibles capaces de generar valor que residen en la propia organización. Es aquel que se queda dentro de la organización cuando los empleados se marchan.

En cuanto a este punto, en el Poder Judicial se debe generar una cultura organizacional que permita que la propia institución se empodere de lo que significa compartir lo que conoce usando para ello la tecnología y aprovechar que mientras sus miembros permanezcan en la organización, su conocimiento y saberes previos puedan ser capturados para formar parte de los repositorios de información de la organización, de tal forma que con su salida no se pierda ese conocimiento implícito que tienen pero que carecen de una cultura de socialización y tampoco la organización tiene mecanismos para capturarlo.

c) El capital relacional está formado por los recursos intangibles capaces de generar valor relacionándolos con el entorno de la empresa: sus clientes, proveedores, sociedad, etc. Son recursos que residen bien en los empleados (capital humano) bien en la propia organización (capital estructural), pero que a efectos conceptuales quedan separados de las dos categorías anteriores por cuanto hacen referencia a relaciones exteriores. El capital relacional es parte del capital intelectual que crea valor en relación con las relaciones exteriores de la empresa.

El Poder Judicial genera un valor público mediante el servicio de justicia que presta y su calidad genera un impacto en su entorno: partes procesales y abogados, colegios de abogados, colegios profesionales, Consejo Nacional de la Magistratura, Ministerio Público, Tribunal Constitucional, Sociedad Civil, etc., que tienen una serie de percepciones del valor público del servicio de justicia. Es aquí donde la gestión del conocimiento en función a sus necesidades debe servir para construir el valor público servicio de justicia, maximizando su resultado con la captura, organización y trasmisión del conocimiento generado en la organización, principalmente a través de la jurisprudencia, plenos jurisdiccionales, pasantías, 
y toda forma de capacitación que se propicie en la institución, para la cual se debe usar las herramientas tecnológicas en el portal del Poder Judicial a efectos que los principales usuarios internos (magistrados) puedan utilizarla, adaptarla, crearla, en la solución de los casos jurídicos que se presentan en la práctica y compartirla, generando retroalimentación entre sus miembros.

Aún cuando se ha mejorado la parte informática con la implementación de nuevas computadoras, sin embargo, dentro de la gestión del conocimiento, la tecnología es un elemento transversal, siendo el eje central del desarrollo la persona, es decir, sus integrantes. Consideramos que un primer intento de gestionar el conocimiento dentro del Poder Judicial se dio por acuerdo de la Sala Plena de la Corte Suprema con la Resolución Administrativa N 062-2007-P-PJ, que aprobó la Directiva N 001-2007-P-CS-PJ, de fecha 22 de febrero de 2007, de "formación de la base de datos, publicación en página web del Poder Judicial y registro estadístico de ejecutorias supremas", cuyo objetivo es identificar los principales criterios establecidos por las salas de la Corte Suprema y orientar las actividades de las instancias de menor jerarquía evitando de esta forma decisiones contradictorias y lograr predictibilidad en las resoluciones judiciales. Se busca generar una línea base del tipo o clase, magnitud y frecuencia de los conflictos jurídicos sociales sometidos a conocimiento de los órganos jurisdiccionales y lograr mayor transparencia en el sistema, estableciéndose en dicha directiva los procedimientos de la constitución de archivo informático de sentencias y autos definitivos.

Igualmente, el Consejo Ejecutivo del Poder Judicial emitió la Resolución Administrativa $\mathrm{N}^{\circ}$ 121-2008-CE-PJ, de fecha 29 de abril que aprueba la Directiva No 009-2008-CE-PJ sobre "Registro sistematizado de las resoluciones judiciales expedidas por los órganos jurisdiccionales de los distritos judiciales y su publicación en la página web del Poder Judicial". Esta última directiva está en proceso de implementación a fin de difundir la jurisprudencia que es una tarea del Centro de Investigaciones Judiciales, para ello se está llevando a cabo un proceso de capacitación del personal responsable de la ejecución de dicha directiva en coordinación con la Gerencia General del Poder Judicial para que se implementen las herramientas tecnológicas para la publicación de las resoluciones judiciales que son construcción de conocimiento jurídico en diversas áreas en base a casos concretos, conocimiento, doctrina, derecho comparado, experiencia, etc. La jurisprudencia es una herramienta clave para que toda la judicatura pueda construir una forma de comunicación entre sus 
integrantes, así como con los usuarios externos como parte del diseño de una política judicial, logrando de esta forma que todos conozcan el pensamiento jurisprudencial que se maneja tanto a nivel de la Corte Suprema como en las 29 Cortes Superiores, y de esta forma que el trabajo de los jueces a nivel nacional no solo sea predecible sino que sea una herramienta para aligerar la forma de resolver los casos sometidos a su competencia, permitiendo manejar la carga procesal existente y se pueda lograr a gran escala, utilizando herramientas tecnológicas (intranet, correo electrónico, groupwise, etc.), la interacción entre los jueces a nivel nacional, de tal manera que se pueda superar las distancias.

La gestión del conocimiento debe ser una herramienta aplicable no solo a nivel jurisprudencial sino también en los Plenos Jurisdiccionales donde deben participar la mayoría de Magistrados, independientemente de su especialidad, puesto que la sociedad actual requiere un magistrado preparado en forma integral, competitivo y eficiente para dar respuestas rápidas frente a las nuevas exigencias de un mundo cambiante. Los magistrados deberían tener acceso a los plenos jurisdiccionales a nivel nacional en tiempo real a través de las videoconferencias en simultáneo y puedan participar en forma activa y aprender a compartir el conocimiento implícito que poseen con el resto de la institución.

Otras formas de compartir el conocimiento podrían darse en las becas de estudios o viajes de capacitación al extranjero de sus miembros, cuyos gastos son asumidos por el Poder Judicial. En estos casos, debe ser política de la institución que los beneficiados deben replicar los conocimientos aprendidos a toda la organización mediante la entrega de una copia del material oficial entregado al Centro de Investigaciones Judiciales para que este lo difunda en un link del portal del Poder Judicial, conferencias, videoconferencias en vivo, a todas las 29 Cortes del país. Debemos considerar que el conocimiento adquirido por los integrantes de la organización es una inversión del Estado y por lo tanto debe socializarse, de esa forma los magistrados estarán en mejores condiciones de resolver los casos sometidos a su competencia mediante una constante actualización y acceso a la información.

Francesc Solé y Martha Olea afirman que la suma de los componentes de las tres categorías decapital intelectual es el capital intelectual dela organización. De la interacción de una categoría con las otras dos se obtiene el capital intelectual más valioso. Concluyen que la gestión del capital intelectual es un concepto más amplio que la gestión del conocimiento. La gestión del 
conocimiento se lleva acabo con el objetivo de adquirir o aumentar el stock de recursos intangibles que crean valor en una organización y por tanto es una parte del concepto más global de gestión de los intangibles ${ }^{10}$.

La gestión de la información y la gestión del conocimiento no son procesos simultáneos. La gestión de la información es previa a la gestión del conocimiento. De hecho, la información se convierte en conocimiento cuando "alguien" la ha contextualizado, deliberadamente o no, de forma que gracias a este proceso de contextualización mejore su capacidad de actuar de forma inteligente (proceso cognitivo) ${ }^{11}$.

Una de las características que presenta la información en el Poder Judicial es que es densa, no solo por el número de personas que demandan tutela jurisdiccional y que el juez debe resolver con conocimiento implícito y explícito, sino porque a su vez cada magistrado representa una fuente permanente de creatividad, y aún cuando la fuente directa es la ley, sin embargo, esta permite espacios de interpretación y creación de derecho. Comparativamente podemos afirmar que, si en el universo tenemos estrellas 10 a la 15 y el ser humano es capaz de establecer relaciones con otros objetos 10 a la 17, entonces podemos sostener que el ser humano tiene la capacidad de crear ideas como estrellas existen en el universo. En consecuencia, el Poder Judicial no debe soslayar la gestión del conocimiento como una herramienta eficaz para el manejo de la complejidad de información que fluye en sus despachos, por el contrario, sus órganos de gobierno deberían incorporarla como política institucional a fin de capturar, crear, innovar, utilizar y compartir todo el conocimiento diseminado en su interior, utilizando complementariamente como elemento transversal a la tecnología para dar respuestas a un mundo cada vez mas cambiante y competitivo, y convertirse en una institución pública inteligente.

\section{OBJETIVOS DE LA GESTIÓN DEL CONOCIMIENTO}

Es importante destacar los objetivos de la gestión del conocimiento para comprender que estos tienen relación y tendrían impacto positivo en el valor público que presta el Poder Judicial. Estos buscan fortalecer para que los agentes obtengan mejores resultados. Se pueden mencionar:

a) Poner en funcionamiento los medios necesarios para conseguir la información y el conocimiento.

b) Administrar el conocimiento organizacional y el aprendizaje organizacional. 
Iris Estela Pacheco Huancas - Aplicación de la gestión

del conocimiento en el Poder Judicial

c) Construir marcos integrados más eficientes.

d) Crear una base tecnológica adecuada al contexto y espacio donde se va a $\operatorname{aplicar}^{12}$.

En el caso del Poder Judicial, tendría que implementarse herramientas tecnológicas de acuerdo a los actores que participan en este proceso y a la naturaleza del servicio que presta.

\section{FACTORES CLAVES DE LA GESTIÓN DEL CONOCIMIENTO}

La gestión del conocimiento se ve enfrentada a una serie de dificultades que provienen del mismo entorno, especialmente de los factores culturales (los individualismos, la falta de una cultura basada en el conocimiento, el aislamiento del entorno y de los integrantes de ese entorno, las orientaciones a corto plazo, etc. $)^{13}$.

Para poner en práctica cualquier tipo de proyecto es necesario tener en cuenta las variables que son influyentes o determinantes en el resultado. Por lo tanto, hay que observar si existe en la organización:

a) Una cultura orientada al conocimiento.

b) Una infraestructura tecnológica de conocimiento.

c) La relación directa entre la gestión del conocimiento y las estrategias de desarrollo.

d) La armonización del lenguaje. Es fundamental, especialmente cuando existen dentro del mismo espacio, culturas, profesiones, ambientes, experiencias y diferentes.

e) Los sistemas de recompensas y estímulos a compartir el conocimiento y a producirlo. Ello neutraliza las barreras que pueden dificultar la gestión del conocimiento.

f) La estructura de conocimiento. Esta debe ser adecuada a los usuarios del sistema. Cada caso va a necesitar contar con un sistema que facilite la dinámica del mismo.

g) Los diversos canales de comunicación del conocimiento. Todos aquellos que produzcan un sentido de confianza y acercamiento entre las personas involucradas.

h) La visualización de las ventajas del sistema ${ }^{14}$.

No debemos olvidar que estamos gestionando personas, cultura y tecnologías. 
Todos los factores mencionados no son ajenos al Poder Judicial, por lo tanto, la gestión del conocimiento no solo es aplicable a las empresas privadas, como algunos equivocadamente sostienen y restringen su campo de aplicación, sino también a las instituciones públicas, y así lo han empezado a entender algunas entidades públicas para incorporarla como política institucional y mejorar el servicio público que prestan.

\section{CAMPO DE APLICACIÓN DE LA GESTIÓN DEL CONOCIMIENTO}

Siendo la naturaleza de la gestión del conocimiento de carácter interdisciplinario y la tecnología un elemento transversal, su campo de aplicación es para todo tipo de organización, sea pública o privada. Si bien es cierto en casi toda la literatura revisada la gestión del conocimiento está orientada a la empresa privada en donde el mercado es el equilibrio, a diferencia de la administración pública donde opera el mercado político que tiene poder para tomar decisiones, se institucionaliza a través de reglamentos y exige transparencia. Son esas prácticas privadas que han logrado resultados positivos las que deben ser recreadas para adaptarse y articularse en organizaciones complejas como las instituciones públicas, en este caso el Poder Judicial, donde el valor público impartición de justicia es un concepto bastante fuerte y un tema de política pública. En el órgano jurisdiccional la gestión del conocimiento debe aplicarse teniendo en cuenta a un país pluricultural y diverso, eliminando la brecha digital que puede existir entre los 29 distritos judiciales del país, a efectos de que todos tengan la oportunidad de acceder a la información a través de herramientas tecnológicas modernas.

Resulta entonces pertinente citar a J. L. Lara quien sostiene que "en una época caracterizada como la era de la información con una altísima tasa de crecimiento tanto en el ritmo como en la profundidad de los cambios, el imperativo es innovar o quedarse atrás. Esta versión aggiornada del "adáptate o muere" darwiniano exige a las organizaciones recrearse permanentemente generando nuevas significaciones en su interacción con el entorno, lo que a su vez recrea el entorno"15. Este mismo autor también nos da la respuesta que la gestión del conocimiento sí se puede aplicar a todo tipo de organizaciones, pero en particular las organizaciones del Estado disponen de una ventaja comparativa sustancial respecto a sus pares del ámbito privado: una estabilidad sensiblemente superior, que proporciona una base de conocimiento institucional sólida sobre la cual establecer las estrategias para elevar los niveles de aptitud recuperando la capacidad dialógica de sus miembros ${ }^{16}$. 
Iris Estela Pacheco Huancas - Aplicación de la gestión

del conocimiento en el Poder Judicial

\section{METODOLOGÍA PARA LA GESTIÓN DEL CONOCIMIENTO}

En un proyecto de gestión del conocimiento, para que tenga éxito tanto en la actividad privada como pública, en este caso específicamente nos referimos al Poder Judicial, el primer referente es identificar a todos los actores involucrados con la iniciativa del proyecto, por ello es importante que el FODA se construya sobre la base de las percepciones de los actores de toda la organización para saber en qué contexto estamos operando. Definitivamente, quien tiene que llevar adelante el proyecto es quien lidera el mismo en la organización.

La metodología en definitiva será diseñada de acuerdo al tipo de organización en la que se quiera aplicar. Esta metodología deberá ser apropiada a la organización judicial con características peculiares. Si bien las tecnologías de la información son importantes como una forma de metodología para la gestión del conocimiento, sin embargo, siempre serán las personas generadoras del conocimiento el elemento central de toda organización.

Al respecto Mela Bosch Jover, citando a Lehay, T. Extrancting Diamonds in the Rough dice: "Veamos entonces cuáles son las metodologías de apoyo que se están usando para los generadores de conocimiento. Se trata de lo que se denomina las buenas prácticas ${ }^{17}$. Bosch sostiene que este es un punto que requiere la formación de las personas que trabajan, la incentivación de su voluntad de intercambio y el establecimiento de hábitos de identificación de los materiales que producen ${ }^{18}$.

Muchas de estas metodologías son muy viejas y ahora cobran especial interés y características propias en el entorno digital de producción intelectual. Tenemos, en primer lugar, las metodologías de presentación de documentos: los informes técnicos, comerciales administrativos y científicos. Es importante que la organización establezca normas comunes para la presentación que contemplen datos en el cuerpo del documento: autoría o protagonistas, estructura de procedencia, datación; en cuanto al contenido: resumen, palabras clave, encabezamiento, así como normas para titulado específico y de citación de otros documentos. En segundo lugar, la identificación de los documentos digitales que tienen dos aspectos: el estado del documento y sus propiedades ${ }^{19}$.

En cuanto al estado o nivel de uso, se utiliza la metodología de organizar árboles de directorio que permitan agrupar los documentos activos con control de versiones, luego los documentos en archivos intermedios, que son de acceso esporádico y, finalmente, los archivos históricos ${ }^{20}$. 
Peluffo y Catalán definen seis etapas ${ }^{21}$ en el ciclo permanente que permite incorporar la gestión del conocimiento como práctica habitual en una organización que administra el conocimiento organizacional como su recurso estratégico más valioso. Estas etapas son:

\section{A. Etapa 1: Diagnóstico inicial de la gestión del conocimiento}

Determina el estado en que se encuentra el sistema de gestión de conocimiento al interior de la organización, con lo cual se van a definir las necesidades de conocimiento y su gestión (tecnología, procesos, personas y valores). Comprende la elaboración de:

\section{a) Mapa de conocimiento organizacional}

La pregunta que interesa responder en relación con el conocimiento organizacional es la siguiente ¿cuánto sabe de lo que sabe? Utilizando la metodología adecuada se configura un diagrama que permite identificar:

- Lo que sabe que sabe: El conocimiento que la organización sabe que conoce.

- Lo que sabe que no sabe: El conocimiento que la organización requiere pero que sabe que no posee.

- Lo que no sabe que sabe: El conocimiento que la organización posee y que no está siendo utilizado.

- Lo que no sabe que no sabe: El conocimiento que la organización ignora que no conoce (pérdida o carencia no visualizada).

\section{a) Diagnóstico de prácticas habituales}

Se investiga los flujos de conocimientos y los procesos que se han implantado para facilitar su administración, desde la fuente (productor o proveedor de conocimientos) hasta el destino (cliente o usuario de conocimientos), considerando especialmente los mecanismos de retroalimentación e intercambio que aseguren un aprendizaje permanente. Permitirá elaborar una matriz de relación que indique de qué manera están alineadas las necesidades reales de conocimientos para efectuar acciones concretas o las expectativas de contar con estos conocimientos, respecto de la utilidad de las fuentes que se están consultando.

\section{c) Evaluación de las capacidades dinámicas de la organización}

La capacidad de absorción del conocimiento y su relación con la innovación en la organización ha permitido a los investigadores desarrollar modelos 
para evaluar estas capacidades dinámicas, en tanto estas permitan absorber e integrar a la organización, información y conocimiento disponible en las redes, permitiéndoles crean nuevas propuestas y soluciones que tienen un impacto en el rendimiento de la organización

\section{B. Etapa 2: Definición de los objetivos del conocimiento}

Todas las iniciativas asociadas a la gestión de conocimiento plantean ciertos objetivos a sus líderes y ciertos plazos de cumplimiento de tales objetivos. En la práctica, los proyectos de gestión de conocimiento se van implementando en etapas sucesivas en las cuales se pretende alcanzar los objetivos globales.

\section{Etapa 3: Producción de conocimiento organizacional}

La generación de conocimiento organizacional representa la base de sustentación de los procesos de aprendizaje organizacional que a su vez permite el desarrollo de las capacidades de adaptación que requieren las organizaciones frente a los cambios en los entornos en los cuales se desenvuelven.

\section{Etapa 4: Almacenaje y actualización}

Se caracteriza por el almacenamiento de los conocimientos previamente codificados, ubicándolos en repositorios desde los cuales los usuarios pueden acceder fácilmente a un conocimiento pertinente y en el momento que lo necesiten.

\section{E. Etapa 5: Circulación y utilización de conocimientos: los usuarios}

Tiene que ver con los espacios de conversación e intercambio adecuados para que se produzca la circulación del conocimiento tácito y explícito de la organización. En conjunto con los espacios de aprendizaje, estos ambientes son los propicios para que los conocimientos puedan fluir de manera ininterrumpida, de manera que se logre el objetivo de la distribución y el uso de tal conocimiento. Ambientes que generen redes de colaboración comunitaria que tiendan a dar respuestas a problemas comunes, la forma más conocida es los sistemas de correo.

\section{F. Etapa 6: Medición de desempeño}

Esta es una fase que está presente periódicamente y su objetivo es determinar en cada uno de los ciclos en que se produce la medición misma, la tendencia 
en los indicadores que se han seleccionado para visualizar de qué forma la gestión de conocimiento está produciendo impactos en los resultados esperados de la organización, sea esta del ámbito privado o público.

En líneas generales, los indicadores deben apuntar a medir la eficiencia y efectividad que se logra en los procesos principales y presentes en la definición de gestión del conocimiento, esto es: generar, compartir y utilizar conocimiento. Estos indicadores se construyen en las siguientes preguntas: a) ¿qué capacidad de generación de conocimiento ha desarrollado la organización a partir de la instalación de las prácticas de gestión de conocimiento?; b) ¿cómo se están compartiendo los conocimientos tácitos y explícitos existentes?, y, c) ¿cuál es la tasa de utilización del conocimiento que está disponible en la organización?

\section{CONCLUSIONES}

a) La mayoría de autores consultados sostienen que no existe duda que la gestión del conocimiento hoy resulta un elemento clave en cualquier tipo de organización que busca ser competitiva en un mundo globalizado. El Poder Judicial no es ajeno a esta realidad organizacional digital de compartición del conocimiento, es más, lo requiere para cambiar su forma de trabajo.

b) Debe tenerse claro que la gestión del conocimiento si bien tiene un elemento transversal que es la tecnología, sin embargo, es la persona el elemento central en la organización, quien es la que genera el flujo de conocimiento que en el caso del Poder Judicial es mediante la jurisprudencia que construyen los jueces y otras formas de captura de conocimiento como plenos jurisdiccionales, pasantías, talleres, cursos de capacitación, congresos, etc., conocimiento que debe ser compartido utilizando la tecnología.

c) La gestión del conocimiento es interdisciplinaria por cuanto no solo se limita a un área del conocimiento sino a toda esa complejidad tanto de las ciencias naturales como sociales y es aplicable a cualquier tipo de organización como sería el Poder judicial, de esta forma capturaría el conocimiento implícito que tienen sus integrantes para convertirlo en patrimonio de la institución, de tal forma que cuando un magistrado se retire de la Judicatura no se lo lleve consigo sin ser aprovechado por la institución.

d) Los factores que influyen en cada organización son la cultura de sus integrantes, la tecnología y el liderazgo de quien conduce el proyecto de gestión de conocimiento, por ello es importante que cuando se asuma 
Iris Estela Pacheco Huancas - Aplicación de la gestión del conocimiento en el Poder Judicial

\section{incorporar a la gestión del conocimiento como eje temático de la reforma del Poder Judicial participen todos los actores involucrados, de tal forma que todos se identifiquen con el mismo y pueda tener éxito.}

1 García Jiménez, Antonio. Organización y gestión del conocimiento en la comunicación. España: Ediciones Trea S. L., 2002, p. 50.

2 Ibídem, p. 51.

3 Peluffo A. Martha Beatriz y Catalán Contreras, Edith. Introducción a la gestión del conocimiento y su aplicación al sector público. Instituto Latinoamericano y del Caribe de Planificación Económica y Social - ILPES. Santiago de Chile. 2002, p. 7, Documento de internet.

4 Collison Chris y Parcell Geoff. La gestión del conocimiento. España: Ediciones Paidós Ibérica S.A., 2003, p. 25.

5 Ibídem, p. 25.

6 Asociación Universitaria Iberoamericana de Postgrado. Ob. Cit., p. 30.

7 Asociación Universitaria Iberoamericana de Postgrado. Gestión del conocimiento, pautas y lineamientos generales. Seminarios y reuniones. Técnicas internacionales, programa de fomento de la formación avanzada. Ediciones AUIP, 2003, p. 31.

8 Ibídem, pp. 27-28.

9 Ibídem, pp. 28-29.

${ }_{10}$ Ibídem, p. 31.

11 Ibídem, p. 30.

12 Peluffo A. Martha Beatriz y Catalán Contreras, Edith. Ob. Cit., pp. 18-19.

13 Ibídem, pp. 18-19.

14 Ibídem, p. 19.

15 Hopkins, José Armando. Gestión del conocimiento (material de lectura). Pontificia Universidad Católica del Perú. Escuela de Graduados. Maestría en Educación. pp. 22-24.

16 Ibídem, p. 24.

17 Bosch Jover, Mela. La gestión del conocimiento en el medio digital: viejos problemas de tratamiento de información y aspectos nuevos. Ciencias de la información. Vol. 33. N 1. Abril, 2002, p. 39. Documento de internet.

18 Ibídem, p. 39.

19 Ibídem.

${ }^{20}$ Ibídem, pp. 39-40.

21 Peluffo A. Martha Beatriz y Catalán Contreras, Edith. Ob. Cit., pp. 58-69 